\title{
The Time Symmetric Initial Value Problem for Black Holes
}

\author{
G. W. GibBONS \\ Department of Applied Mathematics and Theoretical Physics, University of Cambridge, \\ Cambridge, U.K.
}

Received March 14, 1972

\begin{abstract}
The time symmetric initial value problem for black holes is discussed. It is shown that if a solution contains marginally trapped surfaces these correspond to minimal surfaces lying inside the black holes. Such minimal surfaces must have spherical topology. These minimal surfaces are used to obtain lower bounds for the areas of event horizons and upper bounds for the efficiency for radiating gravitational radiation. It is shown that moving black holes closer together reduces the energy available and that a single initially distorted black hole (perhaps formed just after a very assymetric collapse) cannot radiate more than $65 \%$ of its rest mass away. "Wormholes" are also briefly discussed.
\end{abstract}

\section{Introduction}

Recently important advances have been made in the theory of black holes in General Relativity. Hawking [1] has provided a precise definition of a black hole in a weakly asymptotically simple space-time as a connected component of that part of a partial cauchy surface $S$ which is inside the future (past) event horizon, $\dot{J}^{-}\left(\mathscr{I}^{+}\right),\left(\dot{J}^{+}\left(\mathscr{I}^{-}\right)\right)$. He has used this definition to show that in a strongly asymptotically predictable spacetime which contains no "naked singularities" then the area of the boundary of a black hole $\partial B(t)$ must increase (decrease) with time. The reader is referred to Hawking's paper for the definitions of $\dot{J}^{-}\left(\mathscr{I}^{+}\right)$etc. He has applied these results to obtaining upper bounds on the amount of gravitational radiation emitted when charged, rotating black holes initially very far apart fall into one another. To do this he has invoked another crucial assumption - the Carter-Israel conjecture which claims that in any asymptotically flat system containing one or more black holes, the exterior solution will eventually settle down to that of KerrNewman.

The two principal reasons for this belief are:

1. Solutions representing a stationary exterior with no singularities on or outside the event horizon seem to be restricted to the Kerr-Newman family [1]. 
2. In a study of small deviations from the spherically symmetric case, Price [2], Doroshkevich, Zel'dovich and Novikov [3], de la Cruz, Chase and Israel [4] have shown that the exterior system does indeed settle down to the system one expects.

In this paper I wish to extend this work by studying particular partial cauchy surfaces. The aim is threefold.

1. To provide some insight into how restrictive the Hawking efficiency limit is and how it relates to Newtonian ideas of what gravitational energy is available to be radiated.

2. To test, by a means first suggested by Penrose, the hypothesis that in a solution which was initially non singular, any singularities which subsequently develop would not be visible from infinity but would be hidden behind an event horizon. Penrose has called this the assumption of a Cosmic Censor. The idea is to try to show that assuming it one could establish a contradiction with the theorem [5] that the mass of an asymptotically flat system must decrease with time. The Cosmic Censor is discussed in [6].

3. To provide some concrete and easily visualized examples of the rather abstract concepts which it has been necessary to introduce into the theory.

To do this I shall discuss the time-symmetric initial value problem $[7,8]$. That is, instead of studying the whole of Einstein's equations, I restrict myself to considering the constraint equations which the cauchy data for a solution must obey. In the case of a solution containing a surface of time symmetry these constraint equations take on a simple form - the cauchy data reduce to giving the metric of a space-like hypersurface $S$ with normal $t^{\alpha}$ on which the intrinsic Ricci scalar ${ }^{3} R$ is related to the energy density by

$$
{ }^{3} R=16 \pi T_{\alpha \beta} t^{\alpha} t^{\beta} .
$$

Solutions to these equations which represent an arbitrary number of black holes which are momentarily at rest with respect to one another have been given by various authors [7,9]. These solutions have the topology of a number of sheets all joined to another sheet by "necks" or "bridges". If the bridges connect regions on the same sheet they are referred to as "wormholes". Associated with these bridges or wormholes are minimal surfaces - surfaces of least area. These minimal surfaces must lie inside the black holes that these solutions represent. Their area is a lower bound to that of the black hole. In this paper I shall prove that these minimal surfaces must have the topology of a sphere - toroidal minimal surfaces are excluded - and that the available energy of a black hole which is initially very distorted is less than $65 \%$ of its initial rest mass energy. 
If two such black holes collided they could lose at most $75 \%$ of their rest mass energy.

Section I describes the method adopted to achieve 1 and 2 and introduces a new assumption - that of "asymptotic reasonableness". I go on to define minimal surfaces in $S$ and prove that they must, under the conditions of interest in this paper, have spherical topology. In Section II I discuss surfaces of time symmetry. In Section III explicit metrics are used to show that as non rotating black holes are let fall into one another from initially closer positions less energy is available to be radiated away in gravitational radiation. Lower bounds to the area of any surface of an assigned homology class are provided and it is argued that it is unlikely that one could produce a contradiction this way.

In Section IV the method of images is used to discuss wormholes and lower bounds are obtained for their areas. It is shown that the minimal surface in question is a totally "geodesic submanifold" - i.e. its second fundamental form must vanish. The results are used to obtain efficiency limits.

Notation. In this paper I use a signature -+++ and Riemannian tensor convention

$$
\begin{aligned}
R_{b c d}^{a} t^{b} & =t_{; d, c}^{a}-t_{, c ; d}^{a} \\
R_{b d} & =R_{b a d}^{a}
\end{aligned}
$$

I adopt units in which $c=G=1$ (geometric units).

Greek indices run from $0-3$.

Latin lower case indices run from $1-3$.

\section{The Method of Areas}

Given a space-time $\mathscr{M}$ containing a partial cauchy hypersurface $S$ with unit time-like normal $t_{\alpha}$ the cauchy data which it is necessary to specify on $S$ in order to determine the geometry of $\mathscr{M}$ consists of 1) the intrinsic metric, $g_{a b}$, of the surface $S$ which it inherits from $\mathscr{M}$; and 2) the second fundamental form $K_{a b}$ of $S$ which determines how $S$ is embedded in $\mathscr{M}$. The cauchy data has to obey certain constraint or initial value equations. Provided $S$ is "asymptotically flat" it has associated with it an initial mass $M_{i}$. Specifically I shall consider data such that the three metric can be expressed near infinity as

$$
g_{a b}=\delta_{a b}+O\left(\frac{1}{R}\right)
$$

$\delta_{a b}$ is the Kronecker delta. $R$ is an area distance such that a sphere at infinity has an area $4 \pi R^{2}$. In this coordinate system the Ricci tensor of $S$ is given, if $g_{a b}$ obeys certain reasonable differentiability conditions 
(uniform smoothness), by

$$
{ }^{3} R_{a b}=\frac{M_{i}}{R^{3}}\left(3 f_{a} f_{b}-\delta_{a b}\right)+O\left(\frac{1}{R^{4}}\right)
$$

where in spherical polar coordinates

$$
\begin{aligned}
& f_{1}=\cos \phi \sin \theta, \\
& f_{2}=\sin \theta \sin \phi, \\
& f_{3}=\cos \theta .
\end{aligned}
$$

I am assuming that near infinity there may be some electromagnetic field but no ponderable matter. This definition of mass corresponds to the mass of the Riesner-Nordstrom solution and any candidate for the mass of the system as defined on $S$ should reduce to the same expression. Another restriction I shall make on $S$ is that the second fundamental form $K_{a b}=O\left(\frac{1}{R^{2}}\right), S$ does not bend about too much near infinity. If the mass density $T_{\alpha \beta} t^{\alpha} t^{\beta}$ is $O\left(\frac{1}{R^{4}}\right)$ then ${ }^{3} R=O\left(\frac{1}{R^{4}}\right)$ and the Eq. (2) is consistent with the gauss-codazzi equation since $f_{a} f_{a}=1$.

How does this mass relate to the mass defined at future (past) null infinity (Penrose [10])? I shall make the following assumption.

Asymptotic Reasonableness. The limit of the mass defined on a 2-surface on $\mathscr{I}^{+}$as the 2-surface is moved towards the singular point $I^{\circ}$ is the same as the corresponding limit defined on $\mathscr{I}^{-}$and that both of these coincide with that defined on any suitable partial cauchy surface $S$. Whether or not this mass is always positive remains an outstanding question [11]. One would expect a spacetime to be asymptotically reasonable if the system it represents was not radiating gravitational radiation in the infinite past nor will it do so in the infinite future.

I shall now assume that $\mathscr{M}$ contains an event horizon $\dot{J}^{-}\left(\mathscr{I}^{+}\right)$which intersects $S$ in a number of connected components $\partial B_{n}$ which are the boundaries of black holes, and that after some suitable time the black holes have collided and merged to form a final black hole whose exterior solution is that of Kerr Newman with at mass $M_{f}$, charge $e_{f}$ and angular momentum per unit mass $a_{f}$. During this time the total area of the event horizon must increase [1] whilst the mass must decrease [5]. The area of the final event horizon is

$$
A_{f}=4 \pi M_{f}\left(2 M_{f}-e_{f}^{2}+2 M_{f}\left(M_{f}^{2}-a_{f}^{2}-e_{f}^{2}\right)^{\frac{1}{2}}\right)
$$


In particular if it is non rotating and uncharged.

$$
A_{f}=16 \pi M_{f}^{2} .
$$

Now let $16 \pi \alpha M_{i}^{2}$ be a lower bound for the sum of the areas $A_{n}$ of the black holes $B_{n}$. That is, it is known that

$$
A=\sum_{n} A_{n}>\alpha 16 \pi M_{i}^{2} .
$$

We have also

$$
\begin{aligned}
A_{f} & >\sum_{n} A_{n} \\
\frac{M_{f}}{M_{i}} & >\sqrt{\alpha}
\end{aligned}
$$

and the efficiency

$$
\eta=\left(M_{i}-M_{f}\right) M_{i}^{-1}<1-\sqrt{\alpha} .
$$

My aim is to find as high a value of $\alpha$ as possible. In particular if $\alpha$ can be shown to exceed unity the mass of the system has increased we have a contradiction and one of our assumptions must have been incorrect. Defining the available energy as $E=M_{i}-A^{\frac{1}{2}}(16 \pi)^{-\frac{1}{2}}$ I am seeking systems with negative available energy. The idea of looking for initial value hypersurfaces containing black holes which are too large for their mass is due to Penrose [12], and is discussed in more detail in [13].

To find a lower bound for $A$ I look for compact orientable 2-surfaces $T$ which are marginally outer trapped surfaces $[14,17]$. These are space-like 2 surfaces whose future directed outward null normals $l^{x}$ have vanishing convergence $\varrho$ (see [15] for definition of $\varrho$ ). The null hypersurface which has $l^{\alpha}$ as its generators cannot in general be an event horizon because the presence of matter or gravitational radiation will cause the normals to converge and the generators of the event horizon must have positive or zero divergence. It is only in the stationary case that it can be zero [1] and so the two surface $\partial B$ and $T$ must in general be distinct. The generators of the null surface spanned by $T$ cannot extend to $\mathscr{I}^{+}$since there they must have non negative divergence so that $T$ must lie inside the event horizon and hence $T$ lies inside $\partial B$. If $s^{\alpha}$ is the unit spacelike normal to $T$ lying in $S$ and directed outwards $l^{\alpha}$ is given by

$\varrho$ is given by

$$
l^{\alpha}=t^{\alpha}+s^{\alpha}
$$

$$
\varrho=t_{\alpha ; \beta} m^{\alpha} \bar{m}^{\beta}+s_{\alpha ; \beta} m^{\alpha} \bar{m}^{\beta}
$$

where $m^{\alpha}, \bar{m}^{\alpha}$ are complex null vectors orthogonal to $t^{\alpha}, \varrho^{\alpha}$ and normalized so that $m^{\alpha} \bar{m}_{\alpha}=+1$. The first term comes from the second fundamental form, $K_{a b}$ of $S$ regarded as embedded in $\mathscr{M}$ and the second from the 
second fundamental form of $T$ regarded as embedded in $S$. If $K_{a b} m^{a} \bar{m}^{b}$ is zero then $T$ is characterised as being an "extremal surface" lying in $S$. An extremal surface is one for which the first variation of its area is zero. If the second variation is always positive then it is a "minimal surface". Note that in the literature what I have called an extremal surface is often referred to as a minimal surface [16]. If the shear or trace free part of the second fundamental form of $T$ also vanishes it will then be a "totally geodesic submanifold" [16] since only then will geodesics initially lying in the surface remain in it. I shall now prove a useful lemma.

Lemma 1. In any Riemannian space $V$ with a metric which is at least twice differentiable and such that there exists an isometry $\phi: V \rightarrow V$ which leaves fixed the points of a submanifold $W$ then $W$ is a totally geodesic submanifold and hence an extremal surface.

Proof. Assume that the lemma were not true, then there exists a geodesic $\Gamma$ lying initially in $W$ which leaves $W$. The isometry $\phi$ takes this into a new geodesic $\phi \circ \Gamma$. Points of the geodesic not in $W$ are taken to be new positions while those in $W$ are left fixed. Thus there exist two distinct geodesics through a point of $W$ with the same initial tangent, which is impossible.

I shall now prove a theorem concerning compact orientable 2 surfaces lying on a 3 surface $S$.

Theorem I. All compact orientable two surfaces $W$, lying in three surfaces $S$ whose Ricci scalar is non negative, and which are minimal surfaces, are homeomorphic to a two sphere.

Proof. For an extremal surface the second variation of the surface with respect to uniform deformations $d t$ along the normals is given by

where

$$
\frac{d^{2} A}{d t^{2}}=-\int\left({ }^{3} R_{a b} S^{a} S^{b}+2 \Sigma^{2}\right) d A
$$

$$
\Sigma^{2}=\frac{1}{2} \sum_{a b} \Sigma^{a b}
$$

and $\Sigma_{a b}$ is the trace-free part of the second fundamental form of $W$. The gauss-codazzi equation relating the gaussian curvature $K$ of $W$ to the curvature $S$ and the second fundamental form is

$$
2 K={ }^{3} R-2{ }^{3} R_{a b} S^{a} S^{b}-2 \Sigma^{2}+\frac{\Theta^{2}}{2}
$$

$\Theta$ is the trace of the second fundamental form and is zero in this case. Thus

$$
\frac{d^{2} A}{d t^{2}}=\int\left(-\frac{{ }^{3} R}{2}-\Sigma^{2}+K\right) d A .
$$


Now by the Gauss-Bonnet theorem $\int K d A=2 \pi \chi=4 \pi(1-p)$ is a topological invariant of $W \cdot \chi$ is the euler characteristic of the surface and $p$ the "genus" or number of handles it has. For a sphere $p=0$, a torus $p=1$ etc. So that unless $p=0 \frac{d^{2} A}{d t^{2}}$ will be non positive, whence follows the theorem.

The Ricci scalar ${ }^{3} R$ is given by the gauss-codazzi equation relating the intrinsic curvature of $S$ to its embedding.

where

$$
{ }^{3} R=16 \pi T_{\alpha \beta} t^{\alpha} t^{\beta}-\frac{2}{3}\left(K_{a}^{a}\right)^{2}+2 S^{2}
$$

$$
S^{2}=\frac{1}{2} S_{a b} S^{a b}=\frac{1}{2}\left[K_{b}^{a}-\frac{1}{3} \delta_{b}^{a} K_{c}^{c}\right]\left[K_{a}^{b}-\frac{1}{3} \delta_{a}^{b} K_{c}^{c}\right]
$$

is the "shear" of the normals and $K_{a}^{a}$ their expansion. $T_{a \beta} t^{\alpha} t^{\beta}$ is the energy density and so provided the normals are not expanding too fast ${ }^{3} R$ will be non negative. In particular if $K_{a b}$ vanishes the theorem holds and in this case Eq. (8) shows that such a surface is also marginally trapped. This result is a companion result to that of Hawking on the topology of Black Holes [1]. I am grateful to Dr. Hawking for pointing out to me the possibility of such a theorem.

\section{The Time Symmetric Initial Value Problem}

If the spacetime $\mathscr{M}$ possesses a surface of time symmetry $S$ then Lemma 1 shows that its second fundamental form must vanish ( $\phi$ is the time symmetry isometry interchanging past and future). Further if there exists a future event horizon there must also exist a past event horizon and these will intersect on $S$ in $\partial B$. In this case the initial value problem takes on a particularly simple form $[7,8]$. The cauchy data reduce to giving the intrinsic metric of $S-g_{a b}$ and this data must obey the simple constraint equation

$$
{ }^{3} R=16 \pi T_{\alpha \beta} t^{\alpha} t^{\beta} .
$$

A good example of such a solution is the Schwarzschild solution. If we examine the Kruskal extension (Fig. 1) [17] we find two horizons, $\mathscr{N}^{+}$and $\mathscr{N}^{-}$, a surface of time symmetry $S$ and a black hole, with boundary $\partial B$. The surface $S$ consists of two asymptotically flat regions connected by a "throat" or "Einstein-Rosen bridge" [18]. The two regions are isometric and the throat is fixed under the isometry so it is an extremal surface.

The metric of $S$ is given by

$$
d \varsigma^{2}=\frac{d r^{2}}{1-\frac{2 m}{r}}+r^{2}\left(\sin ^{2} \theta d \phi^{2}+d \theta^{2}\right)
$$




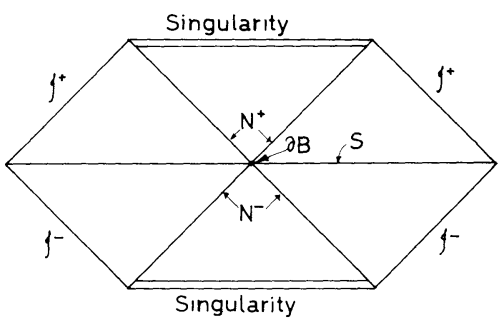

Fig. 1. The maximal analytic extension of the Schwarzschild solution. The diagram represents the $r, t$ manifold, each point represents a 2 sphere. Null lines are at $45^{\circ}$

by the substitution

it becomes

$$
r^{2}=\left(1+\frac{m}{2 \varrho}\right)^{4} \varrho^{2}
$$

$$
d \varsigma^{2}=\left(1+\frac{m}{2 \varrho}\right)^{4}\left[d \varrho^{2}+\varrho^{2}\left(\sin ^{2} \theta d \phi^{2}+d \theta^{2}\right)\right]
$$

where $0<\varrho<\infty$.

A sphere of coordinate radius $\varrho$ has area $4 \pi \varrho^{2}\left(1+\frac{m}{2 \varrho}\right)^{4}$ which has a minimum value of $16 \pi m^{2}$ when $\varrho=\frac{m}{2}$, that is when $r=2 m$ at the Schwarzschild radius. The point $\varrho=0$ has to be excluded from the manifold and so $S$ has the topology of a euclidean 3 space with one point removed i.e. of

$$
\mathbb{R}^{3}-\{0\} .
$$

This example is conformally flat and this lead Misner and Wheeler [7] to consider other solutions of this form. They found solutions representing an arbitrary number of charged non rotating black holes momentarily at rest with respect to one another. From now on I shall restrict myself to the uncharged case since the addition of charge merely increases the available energy without adding any new features unless the charge exceeds the mass (in geometrical units) when as is well known naked singularities enter from the very beginning. The question that the paper is concerned with is the evolution of initially regular cauchy data.

In what follows I shall discuss only the future evolution since in a realistic example it is this part of the solution which would be matched onto a solution representing the collapse of a number of massive bodies which form black holes which are momentarily at rest and then fall into one another. 


\section{III. $N$ Black Holes}

The solutions of Misner and Wheeler [7] are conformally flat

$$
d s^{2}=\Phi^{4} \delta_{a b} d x^{a} d x^{b} .
$$

The vacuum initial value equation reduces to Laplace's equation

$$
\nabla^{2} \Phi=0 .
$$

The only suitable solutions in which the initial surface $S$ is non singular are of the form

$$
\Phi(r)=1+\sum_{C=1}^{C=N} \frac{1}{2} \frac{m_{C}}{\left|r_{C}-r\right|}
$$

where $m_{C}$ are positive constants and $r$ is a position vector in the flat space. The points $\left|r_{C}-r\right|=0$ are excluded from the manifold $S$ which has the topology of $\mathbb{R}^{3}$ with $N$ points removed. This solution is interpreted in the following way. It consists of $N$ sheets joined to an $N+1^{\text {th }}$ sheet via $N$ bridges. Each mass is surrounded by a minimal surface marking the bridge and as one moves through this and towards the points $\left|r_{C}-r\right|=0$ one is moving into a new asymptotically flat region. Associated with each sheet is a mass $m_{C}(1+\chi)$ [9] where

$$
\chi=\sum_{\substack{B=1 \\ B \neq C}}^{B=N} \frac{1}{2} \frac{m_{\beta}}{\left|r_{B}-r_{C}\right|}
$$

The $N+1^{\text {th }}$ sheet has a mass $M_{i}=\sum_{A=1}^{A=N} m_{A}$. The minimal surface around the $c^{\text {th }}$ mass is to be called $T_{c}$ with an area $A_{c}$ (it is also marginally trapped) and there may in addition be further minimal surfaces around any $n$ of them $(1<n<N)$.

As the system evolves it will radiate into each of the $N+1$ asymptotically flat regions. I shall begin by showing:

Theorem II. If $N>1 A_{c}>16 \pi\left(m_{c}\right)^{2}$.

Proof. Consider two metrics $g_{a b}^{\prime}=\left(1+\frac{m_{c}}{\left|r-r_{C}\right|}\right)^{4} \delta_{a b}$ and $g_{a b}=\Phi^{4} \delta_{a b}$. Let $A\left(g^{\prime}, w\right), A(g, w)$ be the area of $W$ evaluating using $g_{a b}^{\prime}$ and $g_{a b}$ respectively then

$$
A(g, w)>A\left(g^{\prime}, w\right)
$$

and let $T_{c}^{\prime}$ be the minimal surface around $m_{C}$ when all the other masses are not present

$$
\begin{aligned}
& A\left(g^{\prime}, T_{c}^{\prime}\right)=16 \pi m_{c}^{2} \\
& A\left(g^{\prime}, T_{c}\right)>A\left(g^{\prime}, T_{c}^{\prime}\right)
\end{aligned}
$$

and the theorem follows. 
From the point of view of an observer in the $c^{\text {th }}$ region the solution represents a single distorted black hole. The energy available for emission is $M_{i}-M_{f}$. From the above result this is bounded by $(1+\chi)^{-1} M_{i}$, thus the possible efficiency $\eta$ for radiation into the $c^{\text {th }}$ sheet is less than $1-(1+\chi)^{-1}$. As the mass points move together the surface $T_{c}$ becomes more and more distorted as $\chi \rightarrow \infty$. The upper bound on the efficiency becomes unity, this is improved on in the next theorem.

Theorem III. Any surface enclosing $R$ mass points has an area $A$ bounded below by

$$
2 \pi\left(\sum_{C=1}^{C=R} m_{c}\right)^{2}
$$

Proof. Consider the second variation of the surface with smallest area of the homology class in question. Its geometry may be discussed in terms of $g_{a b}$ or $\delta_{a b}$, when $\delta_{a b}$ is being used the corresponding quantities will be denoted by a subscript $e$ denoting euclidian. It is required to minimize $\int \Phi^{4} d A_{\mathfrak{e}}$ since

we have

$$
\delta \int d A_{e}=\int J \delta n d A_{e}
$$

$$
J+\frac{4}{\Phi} \frac{\partial \Phi}{\partial n}=0
$$

$J=\left(\frac{1}{R_{1}}+\frac{1}{R_{2}}\right)$ is the mean curvature of the surface and $R_{1}, R_{2}$ the principle radii of curvature, $\frac{\partial \Phi}{\partial n}$ is the normal derivative of $\Phi$. One has

$$
M=\sum_{c=1}^{c=R} m_{c}=-\frac{1}{2 \pi} \int \frac{\partial \Phi}{\partial n} d A_{e}=-\frac{1}{2 \pi} \int \frac{1}{\Phi} \frac{\partial \Phi}{\partial n} \Phi d A_{e} .
$$

Applying Schwartz's inequality one obtains

and again

$$
(2 \pi M)^{2} \leqq \int\left(\frac{1}{\Phi} \frac{\partial \Phi}{\partial n}\right)^{2} d A_{e} \int \Phi^{2} d A_{e}
$$

now

$$
\begin{aligned}
& (2 \pi M)^{2} \leqq \int\left(\frac{1}{\Phi} \frac{\partial \Phi}{\partial n}\right)^{2} d A_{e} \sqrt{\left(\int \Phi^{4} d A_{e}\right)} \sqrt{\left(\int d A_{e}\right)} \\
& (2 \pi M)^{2} \leqq \int\left(\frac{1}{\Phi} \frac{\partial \Phi}{\partial n}\right)^{2} d A_{e} \sqrt{A} \sqrt{A_{e}}
\end{aligned}
$$

$$
\frac{d^{2} A}{d t^{2}}=\int\left(K-\Sigma^{2}\right) d A
$$


$\int K d A=\int K_{e} d A_{e}$ since $\int K d A$ is a topological invariant also

so

$$
\Phi^{4} \Sigma^{2}=\Sigma_{e}^{2}=\frac{1}{4}\left(\frac{1}{R_{1}}-\frac{1}{R_{2}}\right)^{2}
$$

$$
\frac{d^{2} A}{d t^{2}}=\int\left(K_{e}-\Sigma_{e}^{2}\right) d A_{e}
$$

It is also true that $\Sigma_{e}^{2}=\frac{1}{4} J^{2}-K_{e} ; J=-\frac{4}{\Phi} \frac{\partial \Phi}{\partial n}$

so

$$
\frac{d^{2} A}{d t^{2}}=\int 2 K_{e} d A_{e}-4 \int\left(\frac{1}{\Phi} \frac{\partial \Phi}{\partial n}\right)^{2} d A_{e}
$$

$$
\frac{d^{2} A}{d t^{2}}<8 \pi-\frac{4(2 \pi M)^{2}}{\sqrt{A} \sqrt{A_{e}}}
$$

$\frac{d^{2} A}{d t^{2}}$ must be positive therefore $\sqrt{A} \sqrt{A_{e}}>2 \pi M^{2}$

$$
A>2 \pi M^{2} \text {. }
$$

Now consider the case of two black holes of equal mass. When they are far apart there will be just two minimal surfaces of area $>16 \pi \mathrm{m}^{2}$. As they are moved closer together the area will increase and there will be less and less available energy - as one would expect on Newtonian grounds. There is a danger that $\alpha$ will exceed unity. However as they are moved closer a third minimal surface will form around both once the euclidean distance apart $d$ is less than $1.59 \mathrm{~m}$ (Brill and Lindquist [9]).

This area is bounded below by $2 \pi M_{i}^{2}$. It is also less than the sum of the two areas enclosed, as can be seen from the following argument. Join the two inside surfaces by a very narrow pipe (see Fig. 2). This can be made of negligible area. The surface then $A_{1}+A_{2}<A_{3}$, since $A_{3}$ is by definition a minimal surface. Thus if the Cosmic Censor Principle holds one would expect that before the two individual surfaces become

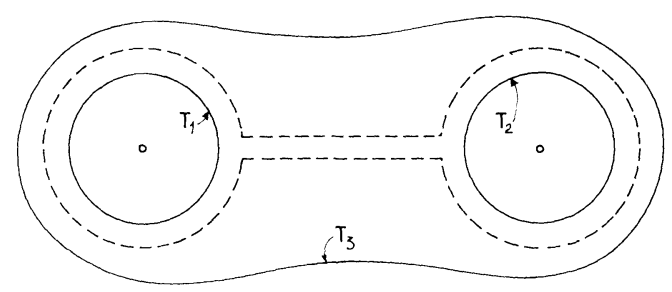

Fig. 2. Two minimal surfaces surrounded by a third. The 2 points represent the asymptotically flat regions on the other side of the Einstein-Rosen bridge. The dotted surface is obtained by continuously deforming $T_{3}$ 
too large the third surface forms around both and saves the day. I have not been able to show this completely using analytic methods but I feel that the following result lends some credence to this belief.

Theorem IV. For the above system the available energy is positive for $d>(\sqrt{2}-1)^{-1} m=2.48 \mathrm{~m}$. Two lemmas are required.

Lemma 2. If $A_{t}$ is a one parameter family of surfaces $t \in(a, b)$ and $A$ the area of the smallest minimal surface of this homology class and

$$
\begin{aligned}
A_{t} & <f(t) \\
A & <\min _{(a, b)} f(t) .
\end{aligned}
$$

The proof is obvious.

Lemma 3. If

$$
\begin{array}{rlrl}
f(t) & =t^{2}\left(\alpha+\frac{\beta}{t}\right)^{4} & & t \in(0, a) \\
\min f(t) & =2^{4} \alpha^{2} \beta^{2} & \text { if } & 0<\frac{\beta}{\alpha}<a \\
\min f(t) & =a^{2}\left(\alpha+\frac{\beta}{a^{2}}\right)^{4} \quad \text { if } & 0<a<\frac{\beta}{\alpha} .
\end{array}
$$

Again the proof is easy.

Proof of Theorem IV. For our one parameter family chose euclidean spheres of radius $r$ about one of the two mass points. The minimal surface about it must lie in its own half space by symmetry.

Thus

$$
\begin{aligned}
& A_{r}=\int\left(1+\frac{m}{2 r}+\chi\right)^{4} d A_{e}<\int\left(1+\frac{m}{2 r}+\frac{m}{d}\right)^{4} d A_{e} \\
& A_{r}<4 \pi r^{2}\left(1+\frac{m}{2 r}+\frac{m}{d}\right)^{4} .
\end{aligned}
$$

Now use Lemmas 2 and 3 to show that then

provided

$$
A<\left(1+\frac{m}{d}\right)^{2} 16 \pi m^{2}
$$

$$
\frac{m}{2}\left(1+\frac{m}{d}\right)^{-1}<\frac{d}{2}
$$

which will always be true. Thus $\alpha=\frac{1}{2}\left(1+\frac{m}{d}\right)^{2}$

which is less than unity for $d<(\sqrt{2}-1)^{-1} m$ whence the result follows. 
It is also possible to apply theorem II to the radiation into the $c^{\text {th }}$ throat mentioned earlier. From the point of view of its infinity the minimal surface $T_{c}$ surrounds all the other points and so its available energy will be $<(1-1 / \sqrt{8}) M_{i}$ which is less than $65 \%$ of the initial mass. This situation might arise when a black hole formed during an extremely assymetric collapse (with no net angular momentum however). Initially the black hole is very distorted but it relaxes back to a spherical state and in the process it can radiate no more than $65 \%$ of its rest mass in gravitational radiation. In practice it will probably radiate much less.

Obviously one can proceed to more complicated situations but as with this case it is most likely that one would need to have recourse to numerical methods.

\section{Wormholes}

Misner $[19,20]$ has generalized the solutions described above to obtain metrics representing the initial conditions for 'wormholes'. A wormhole arises when two separate regions on the same sheet are joined together. The surface on which this join occurs will in general be a minimal surface. Misner's method is one of successive images. He retains the form $d s^{2}=\Phi^{4} \delta_{a l} d x^{a} d x^{l}$ and starts with one of the solutions described above. Then he surrounds each mass point by a sphere and then obtains the image mass of each external mass which will lie inside the sphere by a process of inversion. Then he repeats the process to each mass point in turn, thus obtaining an infinite sequence of images. He shows that the process will converge and by a familiar property of Laplace's equation the potential so obtained will also be a solution. The result of this process is that inside each sphere is a region which is isometric to the exterior of each sphere - the isometry being a sort of inversion. Now one can identify spheres in a suitable way to obtain wormholes or other rather similar structures. In the case of just two spheres he has obtained an explicit form for the metric [19]. For more details the reader is referred to Misner's papers and also to Lindquist [21] who has obtained the charged case.

In this section I shall make two remarks on Misner's method and then obtain an improved lower bound on the area of the minimal surfaces.

1. By Lemma 1 the spheres are totally geodesic submanifolds and hence minimal surfaces.

2. The method can only work for spheres because if a suitable isometry exists $\Sigma^{2}=0$ which implies $\Sigma_{e}^{2}=0$ which means that $T_{e}$ is a euclidean sphere [22].

I shall now use the fact that $\Sigma_{e}^{2}=0$ to give a lower bound on the area of the surface. Because of the repeated inversion Theorem II no longer applies. 
Theorem V. Define the mass of the minimal surface, considered as immersed in the complete euclidean three-space as

Then

$$
m=-\frac{1}{2 \pi} \int \frac{\partial \Phi}{\partial n} d A_{e} .
$$

Proof.

$$
A>4 \pi m^{2}
$$

$$
J+\frac{4}{\Phi} \frac{\partial \Phi}{\partial n}=0 \quad \text { and } \quad J=\frac{2}{r}
$$

when $r$ is the euclidean radius of the sphere.

by Schwartz inequality

$$
\begin{gathered}
2 \pi m=\frac{1}{4} \int \Phi J d A_{e}=\frac{1}{2 r} \int \Phi d A_{e} \\
\int \Phi d A_{e} \leqq A^{\frac{1}{4}} A_{e}^{\frac{3}{4}}
\end{gathered}
$$

$$
2 \pi m \leqq \frac{1}{2 r} A^{\frac{1}{4}} A_{e}^{\frac{3}{3}}<A^{\frac{1}{2}} \sqrt{\pi}
$$

thus $A>4 \pi m^{2}$ since $A>A_{e}$.

Now consider the coalescence of a single wormhole. From the point of view of an observer far away from the wormhole it will look like either 1) two small black holes or 2) one large black hole. It is only when the observer investigates the insides of the black holes that he can tell that in both cases the interiors are connected and hence they should both be referred to as a single black hole within Hawking's definition. However, although there is one black hole present, the intersection of the event horizon, if it is present, with $S$, can consist either of two disjoint surfaces $\partial B_{1}$ and $\partial B_{2}$ both homologous with the minimal surface $T$ and in a loose sense situated at either end of the wormhole; or, there may be just one connected component of the intersection of the event horizon with $S, \partial B_{3}$. In this case it will not be homologous with $T$. These two possibilities are illustrated in Fig. 3. One would expect the first case to hold if the "black holes" are far apart compared with their mass and the second to be the case if they are close together. In the second case one would expect a third minimal surface $T_{3}$ to have formed. The mass of the system $M_{i}$ as seen far away will be $2 m$. If the system collapses and ultimately settles down to a schwarzschild solution with mass $M_{f}$ one has in case 1, using theorem $\mathrm{V}$

$$
16 \pi M_{f}^{2}>2 A>8 \pi m^{2}>2 \pi\left(M_{i}\right)^{2}
$$

and so the efficiency is less than $65 \%$. This is rather better than the $75 \%$ limit one obtains by applying theorem III directly. If one has to treat 




CASE ।

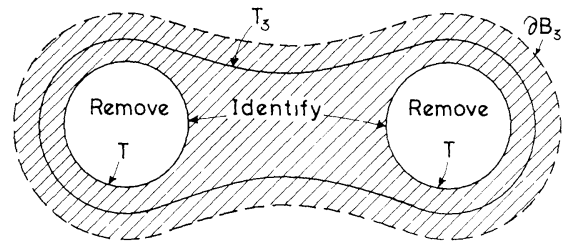

CASE 2

Fig. 3. Possible topologies of a wormhole. Since the system is symmetric about an axis joining the centre of the spheres, each point in the diagram represents a semi-circle. The shaded region represents the interior of a single black hole

case 2 one can apply theorem III to $T_{3}$. This provides the same limit which shows that the available energy of a wormhole is always less than $65 \%$.

\section{Discussion}

In this paper I have discussed the use of trapped and marginally trapped surfaces in the investigation of the interaction of black holes. I have shown that in the cases considered (the time symmetric problem) marginally trapped surfaces must be homeomorphic to a sphere and I have placed lower bounds on their available energy and shown that as black holes are moved closer together the available energy decreases. I have argued that it is unlikely that one could use this method of Penrose to provide a contradiction to the Cosmic Censor assumption.

To do this I have restricted the discussion to non rotating black holes where the initial surface is conformally flat. The second restriction is probably not very important since it corresponds to there being no transverse gravitational waves in the A.D. and M. formalism of General Relativity [23]. The first restriction is however rather more important. It is known that for $a>m$ the Kerr solution contains a naked singularity. The question that naturally arises is this: given a number of black holes with large orbital and spin angular momentum (but such that for each individually $a<m$ ) which fall into one another, can they always radiate away enough of their angular momentum in the form of gravitational radiation to avoid forming a rotating black hole with $a>m$ ? This problem is the major obstacle to a complete understanding of the exterior dynamics of black holes.

Throughout this work I have benefitted greatly from conversations with the members of Cambridge Relativity group and most especially from the interest, encouragement and advice of my supervisor, Dr. S. W. Hawking. I am also most grateful to Professor R. Penrose for helpful discussions and for suggesting the use of the time symmetric initial value problem in this work. 


\section{References}

1. Hawking, S. W.: Commun. math. Phys. 25, 152 (1972).

2. Price, R.: Phys. Rev. To appear.

3. Doroshkevitch,A.G., Zel'dovich, Ya.B., Novikov,I.D.. Zh. Eksp. Teor. Fiz. 49, 170 (1965). English translation in Sov. Phys. J.E.T.P. 22, 122 (1966).

4. de la Cruz, V., Chase, J.E., Israel, W.: Phys. Rev. Letters 24, 423 (1970).

5. Bondi, H., Van der Burg, M. G. J., Metzner, A. W.: Proc. Roy. Soc. A 269, 21 (1962).

6. Penrose, R.: La Rivista del Nuovo Cimento 1 Numero Speciale 252 (1969).

7. Misner, C. W., Wheeler, J. A.: Ann. Phys. 2, 525 (1957).

8. Brill, D. R.: Ann. Phys. 7, 466 (1959).

9. Brill, D. R., Lindquist, R.: Phys. Rev. 131, 471 (1963).

10. Penrose, R.: Phys. Rev. Letters 10, 66 (1963).

11. Robinson, D., Winnicour,J.: J. Math. Phys. 12, 995 (1971).

12. Penrose, R.: Seminar given at Cambridge University (1971).

13. Gibbons, G.W., Penrose, R.: To be published.

14. Penrose, R.: Phys. Rev. Letters 14, 57 (1965).

15. Newman, E., Penrose, R.: J. Math. Phys. 3, 566 (1962).

16. Eisenhart, L.P.: Riemannian geometry, Princeton 1964.

17. Penrose, R.: Article in Batelle recontres (C. De Witt and J. A. Wheeler eds.) New York: W. A. Benjamin, 1968.

18. Einstein, A., Rosen, N.: Phys. Rev. 48, 73 (1935).

19. Misner, C. W.: Phys. Rev. 118, 1110 (1960).

20. Misner, C. W.: Ann. Phys. 24, 102 (1963).

21. Lindquist, R.: J. Math. Phys. 4, 938 (1963).

22. Willmore, T.J.: Differential geometry. Oxford 1959.

23. Arnowitt, R., Deser, S., Misner, C. W.: Ann. Phys. 11, 116 (1960).

G. W. Gibbons

Dept. of Applied Mathematics

and Theoretical Physics

University of Cambridge

Silver Street

Cambridge, England 